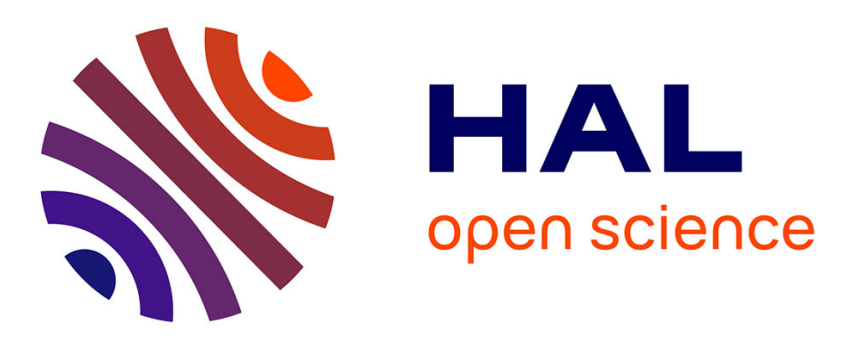

\title{
Identification of an isometric transformation of the standard brownian sheet
}

\author{
Serge Cohen, Xavier Guyon, Olivier Perrin, Monique Pontier
}

\section{To cite this version:}

Serge Cohen, Xavier Guyon, Olivier Perrin, Monique Pontier. Identification of an isometric transformation of the standard brownian sheet. Journal of Statistical Planning and Inference, 2006, 136 (4), pp.1317-1330. 10.1016/j.jspi.2004.09.012 . hal-00271988

\section{HAL Id: hal-00271988 \\ https://hal.science/hal-00271988}

Submitted on 10 Apr 2008

HAL is a multi-disciplinary open access archive for the deposit and dissemination of scientific research documents, whether they are published or not. The documents may come from teaching and research institutions in France or abroad, or from public or private research centers.
L'archive ouverte pluridisciplinaire HAL, est destinée au dépôt et à la diffusion de documents scientifiques de niveau recherche, publiés ou non, émanant des établissements d'enseignement et de recherche français ou étrangers, des laboratoires publics ou privés. 


\title{
Identification of an isometric transformation of the standard Brownian sheet
}

\author{
Serge Cohen* Xavier Guyon, Olivier Perrin ${ }^{\ddagger}$ and Monique Pontier*
}

November 14, 2003

\begin{abstract}
We use quadratic variations to identify almost surely the coordinate system where the standard Brownian sheet is defined. This identification is carried out with the help of an algorithmic-like procedure.
\end{abstract}

keywords: coordinate system; estimation; quadratic variation AMS 2000 SUBJECT CLASSIFICATION: PRIMARY 60J65, 62H12, 62M40

SECONDARY 60G15, 62M 30

\section{Introduction}

Random fields, or spatial processes, are useful for modeling spatial phenomenon like environmental fields including, for example, rain fall, acid precipitation, air pollution, hydrology, geophysics and oceanography, and medical images ( $c f$. [12] and its bibliography).

The analysis, modeling and estimation, of the spatial covariance structure underlying spatial processes has been the subject of increasing research in recent years. Knowledge of the spatial covariance of a random field is fundamental in spatial estimation or kriging and in the design of optimal monitoring networks. As outlined in [11], particularly important is the fact that the spatial covariance structures underlying multi-parameter processes are almost always non-stationary, neither isotropic, over the spatial scales of interest.

In practice, when we model a non-stationary or/and a non-isotropic spatial phenomenon with a random field, the coordinate system where the latter is defined cannot be chosen arbitrarily. In this case, the identification of the coordinate system is as important as the estimation of the parameters of the random field itself. In this paper, the standard Brownian sheet is an illustration of random fields whose definition depends on the coordinate system. We propose

\footnotetext{
${ }^{*}$ LSP, Université Paul Sabatier, 118 route de Narbonne, 31062 Toulouse Cedex 04, France, E-mail: scohen@lsp.ups-tlse.fr and pontier@lsp.ups-tlse.fr

${ }^{\dagger}$ SAMOS, Université Paris I, 90, rue de Tolbiac, 75634 Paris Cedex 13, France, E-mail: guyon@univ-paris1.fr

${ }^{\ddagger}$ GREMAQ, Université Toulouse 1 and LEERNA, INRA, 21, allée de Brienne, 31000 Toulouse, France, E-mail: perrin@cict.fr
} 
an estimation of the coordinate system, where the Brownian sheet is observed, by using quadratic variations. In a first step, we identify the quadrant where the Brownian sheet is defined. Then we give a practical procedure to estimate the coordinate system corresponding to this quadrant.

The quadratic variations are first introduced by Lévy [9] who shows that if $B$ is the standard Brownian process on $[0,1]$, then almost surely its quadratic variation on $[0,1]$ converges to 1 . Baxter [2] and further Gladyshev [4] generalize this result to a large class of Gaussian processes. Guyon and León [6] introduce the $H$-variations for stationary Gaussian processes, a generalization of these quadratic variations. They study the convergence in distribution of the $\mathrm{H}$ variations, suitably normalized.

For Gaussian process $Z$ with stationary increments, Istas and Lang [7] define general quadratic variations, substituting a general discrete difference operator to the simple difference $Z(k / n)-Z((k-1) / n)$. They use these quadratic variations to estimate the Hölder index of a process. The generalization of quadratic variations for stationary Gaussian fields indexed by $\mathbb{R}^{2}$ is studied in Guyon [5] as well as in León and Ortega [8]. Another generalization for nonstationary Gaussian processes over general index spaces and quadratic variations along curves is done in Adler and Pyke [1].

The paper is structured as follows. Section 2 sets up notations, assumptions and definitions. Section 3 describes the quadratic variations and their asymptotic properties. In Section 4, these quadratic variations are combined to propose almost sure consistent estimators of the parameters of the isometric transformation. In Section 5, simulations evaluate the performance of these estimators. Finally, Section 6 discusses one extension of the present work for future research.

\section{Notations, assumptions and definitions}

First note that all angles we are dealing with are oriented in the trigonometric sense. Let $W=\left\{W(x, y),(x, y) \in \mathbb{R}^{2}\right\}$ be the standard Brownian sheet that is a centered Gaussian process indexed by $\mathbb{R}^{2}$ with a covariance function given by

$$
E\left(W\left(x_{1}, y_{1}\right) W\left(x_{2}, y_{2}\right)\right)=\frac{1}{4}\left(\left|x_{1}\right|+\left|x_{2}\right|-\left|x_{1}-x_{2}\right|\right)\left(\left|y_{1}\right|+\left|y_{2}\right|-\left|y_{1}-y_{2}\right|\right) .
$$

This definition is given in a certain coordinate system, say $\left(O^{\prime}, \mathcal{X}, \mathcal{Y}\right)$. Suppose that instead of $W(x, y)$, we observe $Y_{\theta, a, b}(u, v)=W(x(u, v), y(u, v))$ where

$$
\left\{\begin{array}{l}
x(u, v)=(u-a) \cos (\theta)+(v-b) \sin (\theta) \\
y(u, v)=-(u-a) \sin (\theta)+(v-b) \cos (\theta),
\end{array}\right.
$$

so that the covariance function of $Y_{\theta, a, b}$ is given by

$$
\begin{aligned}
& E\left(Y_{\theta, a, b}\left(u_{1}, v_{1}\right) Y_{\theta, a, b}\left(u_{2}, v_{2}\right)\right) \\
& =\frac{1}{4}\left(\left|x\left(u_{1}, v_{1}\right)\right|+\left|x\left(u_{2}, v_{2}\right)\right|-\left|x\left(u_{1}, v_{1}\right)-x\left(u_{2}, v_{2}\right)\right|\right) \\
& \quad \times\left(\left|y\left(u_{1}, v_{1}\right)\right|+\left|y\left(u_{2}, v_{2}\right)\right|-\left|y\left(u_{1}, v_{1}\right)-y\left(u_{2}, v_{2}\right)\right|\right) .
\end{aligned}
$$


The random field $Y_{\theta, a, b}$ can be viewed as a generalization of the standard Brownian sheet, the latter being $Y_{0,0,0}$. In practice, this means that if we want to model a phenomenon with the help of the standard Brownian sheet for which we do not know the coordinate system where it is defined, we must identify the unknown parameters $\theta, a$ and $b$.

As illustrated by Figure 1, the standard Brownian sheet $W$ is defined in the unknown coordinate system $\left(O^{\prime}, \mathcal{X}, \mathcal{Y}\right)$, the canonical coordinate system of the standard Brownian sheet, and is observed through $Y_{\theta, a, b}$ in an arbitrarily chosen coordinate system $(O, \mathcal{U}, \mathcal{V})$. The transformation from $(O, \mathcal{U}, \mathcal{V})$ to $\left(O^{\prime}, \mathcal{X}, \mathcal{Y}\right)$ is a rotation through an angle $\theta$ about the origin $O$ followed by a translation of vector $(a, b)$, so that it is a direct isometric transformation.

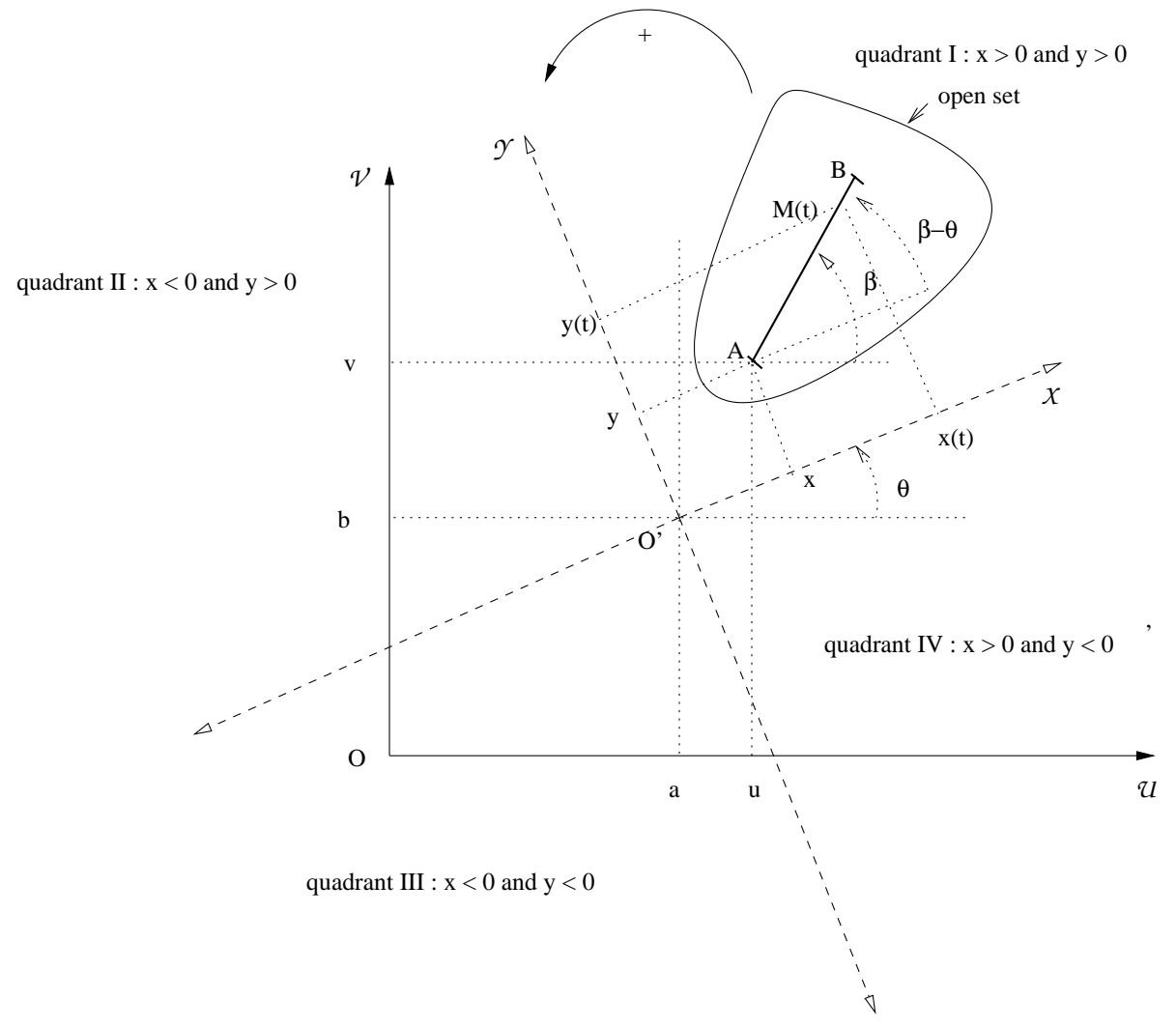

Figure 1: Isometric transformation of the coordinate system

In this paper, we are interested in the estimation of the parameter angle $\theta$ together with the parameters of the translation $a$ and $b$, from one realization of the random field $Y_{\theta, a, b}$ observed on some open subset in the coordinate system $(O, \mathcal{U}, \mathcal{V})$. In fact, because $Y_{\theta, a, b}$ is almost surely equal to 0 on the axes of the canonical coordinate system, we assume that $Y_{\theta, a, b}$ is observed in an arbitrary non vacuous open set of $\mathbb{R}^{2}$ which does not intersect any axis of the canonical coordinate system. The estimation is carried out with the help of quadratic variations. Results from Baxter (1956) and its generalizations in Perrin (1999) are widely used. 


\section{Quadratic variations}

In this paper, we only consider quadratic variations along segments, like the segment $[A, B]$ of length $L>0$. This segment is included in the open set previously mentioned and is represented in Figure 1, where $A$ is the point of coordinates $(u, v)$ in $(O, \mathcal{U}, \mathcal{V})$. Let $\beta$ denote the angle between $\overrightarrow{O u}$ and $\overrightarrow{A B}$. Each point $M(t)=(x(t), y(t))$ belonging to $[A, B]$, where $(x(t), y(t))$ are the coordinates of $M(t)$ in $\left(O^{\prime}, \mathcal{X}, \mathcal{Y}\right)$, has the following parametric representation in $\left(O^{\prime}, \mathcal{X}, \mathcal{Y}\right)$, for $t \in[0,1]$

$$
\begin{gathered}
\overrightarrow{O^{\prime} M}(t)=\overrightarrow{O^{\prime} A+t \overrightarrow{A B}} \\
\Longleftrightarrow\left\{\begin{array}{l}
x(t)=(u-a) \cos (\theta)+(v-b) \sin (\theta)+L t \cos (\beta-\theta) \\
y(t)=-(u-a) \sin (\theta)+(v-b) \cos (\theta)+L t \sin (\beta-\theta) .
\end{array}\right.
\end{gathered}
$$

Without restriction, we may impose $\theta \in\left[0, \frac{\pi}{2}[\right.$. Indeed, the canonical coordinate system $\left(O^{\prime}, \mathcal{X}, \mathcal{Y}\right)$ is at most at an angle $\frac{\pi}{2}$ of the current coordinate system $(O, \mathcal{U}, \mathcal{V})$. Unlike $\theta$, the angle $\beta$ is a parameter under our control, like $L, u$ and $v$, and we choose $\beta$ in $\left[0, \frac{\pi}{2}\right]$. Thus the process $Z$ indexed by $[0,1]$ and defined by

$$
Z(t)=W(x(t), y(t))
$$

is the restriction of $W$ to the segment $[A, B]$.

We set

$$
\left\{\begin{array}{l}
x=x(0)=(u-a) \cos (\theta)+(v-b) \sin (\theta) \\
y=y(0)=-(u-a) \sin (\theta)+(v-b) \cos (\theta)
\end{array}\right.
$$

where $(x, y)$ are the coordinates of $A$ in $\left(O^{\prime}, \mathcal{X}, \mathcal{Y}\right)$.

In the next Paragraph, we define the quadratic variations along the segment $[A, B]$. As we will see in Paragraph 3.2, the behavior of these quadratic variations will depend on the quadrant of $\left(O^{\prime}, \mathcal{X}, \mathcal{Y}\right)$ in which the segment $[A, B]$ is defined.

\subsection{Results in the positive quadrant}

In a first step, we assume that $x$ and $y$ are strictly positive. This means that the segment $[A, B]$ is included in the positive quadrant (quadrant I of Figure 1) of the canonical coordinate system $\left(O^{\prime}, \mathcal{X}, \mathcal{Y}\right)$ and that it does not meet its axes. In the following Paragraph, we will consider the three other cases $(x<0$ and $y>0$ (quadrant II), $x<0$ and $y<0$ (quadrant III), and $x>0$ and $y<0$ (quadrant IV), i.e. corresponding to the respective three other quadrants.

Let $n$ be a positive integer. We set for $k=1,2, \ldots, n$

$$
\Delta Z_{k}=Z(k / n)-Z((k-1) / n)
$$

Let $\Pi_{n}(1)=\left\{0, \frac{1}{n}, \frac{2}{n}, \ldots, \frac{n-1}{n}, 1\right\}$ be the regular partition of $[0,1]$ at constant scale $1 / n$. We denote using $[n t]$ the greatest integer smaller than or equal to 
$n t$. For $t \in[0,1]$, we define the quadratic variations $V_{n}(t ; \beta, u, v, L)$ of $Z$ along $\Pi_{n}(t)=\left\{0, \frac{1}{n}, \frac{2}{n}, \ldots, \frac{[n t]}{n}\right\}$ as follows

$$
V_{n}(t ; \beta, u, v, L)=\sum_{k=1}^{[n t]}\left(\Delta Z_{k}\right)^{2} .
$$

When $[n t]=0$, we set $\sum_{k=1}^{0}\left(\Delta Z_{k}\right)^{2}=0$.

Before giving the main results concerning the asymptotic properties of $V_{n}(t ; \beta, u, v, L)$ as $n \rightarrow \infty$ that follow from Perrin [10], we must check that the covariance function $r\left(t, t^{\prime}\right)=E\left(Z(t) Z\left(t^{\prime}\right)\right)$ of $Z$ is continuous in $[0,1]^{2}$ and has second derivatives which are uniformly bounded for $t \neq t^{\prime}$.

For positive $x_{1}, y_{1}, x_{2}$ and $y_{2}$, the definition (1) can be written as

$$
E\left(W\left(x_{1}, y_{1}\right) W\left(x_{2}, y_{2}\right)\right)=\left(x_{1} \wedge x_{2}\right)\left(y_{1} \wedge y_{2}\right),
$$

so that we deduce from the definition $(2)$, for all $t, t^{\prime}$ in $[0,1]$

$$
\begin{aligned}
r\left(t, t^{\prime}\right)=E\left(Z(t) Z\left(t^{\prime}\right)\right)=(x+L t & \cos (\beta-\theta)) \wedge\left(x+L t^{\prime} \cos (\beta-\theta)\right) \\
& \times(y+L t \sin (\beta-\theta)) \wedge\left(y+L t^{\prime} \sin (\beta-\theta)\right) .
\end{aligned}
$$

We denote using $r^{\left(m, m^{\prime}\right)}\left(t, t^{\prime}\right)$ the $m, m^{\prime}$-partial derivative of $r$ with respect to $t$ and $t^{\prime}$. Depending on the sign of $\beta-\theta$ we have to consider two situations

- Situation 1: $0 \leq \beta-\theta \leq \frac{\pi}{2}$

- If $t^{\prime}>t$, then $r\left(t, t^{\prime}\right)=(x+L t \cos (\beta-\theta))(y+L t \sin (\beta-\theta))$ so that

$$
\left\{\begin{array}{l}
r^{(2,0)}\left(t, t^{\prime}\right)=L^{2} \sin (2(\beta-\theta)) \\
r^{(1,1)}\left(t, t^{\prime}\right)=0 \\
r^{(0,2)}\left(t, t^{\prime}\right)=0
\end{array}\right.
$$

and

$$
r^{(0,1)}\left(t, t^{\prime}\right)=0 .
$$

- If $t^{\prime}<t$, then $r\left(t, t^{\prime}\right)=\left(x+L t^{\prime} \cos (\beta-\theta)\right)\left(y+L t^{\prime} \sin (\beta-\theta)\right)$ so that

$$
\left\{\begin{array}{l}
r^{(2,0)}\left(t, t^{\prime}\right)=0 \\
r^{(1,1)}\left(t, t^{\prime}\right)=0 \\
r^{(0,2)}\left(t, t^{\prime}\right)=L^{2} \sin (2(\beta-\theta)),
\end{array}\right.
$$

and

$$
\begin{aligned}
r^{(0,1)}\left(t, t^{\prime}\right) & =L \cos (\beta-\theta)\left(y+L t^{\prime} \sin (\beta-\theta)\right)+L \sin (\beta-\theta)\left(x+L t^{\prime} \cos (\beta-\theta)\right) \\
& =L \cos (\beta-\theta) y+L \sin (\beta-\theta) x+L^{2} t^{\prime} \sin (2(\beta-\theta)) \\
& =L(u-a) \sin (\alpha-\theta)+L(v-b) \cos (\alpha-\theta)+L^{2} t^{\prime} \sin (2(\beta-\theta)) .
\end{aligned}
$$

- Situation 2: $-\frac{\pi}{2} \leq \beta-\theta \leq 0$ 
- If $t^{\prime}>t$, then $r\left(t, t^{\prime}\right)=(x+L t \cos (\beta-\theta))\left(y+L t^{\prime} \sin (\beta-\theta)\right)$ so that

$$
\left\{\begin{array}{l}
r^{(2,0)}\left(t, t^{\prime}\right)=0 \\
r^{(1,1)}\left(t, t^{\prime}\right)=\frac{L^{2} \sin (2(\beta-\theta))}{2} \\
r^{(0,2)}\left(t, t^{\prime}\right)=0,
\end{array}\right.
$$

and

$$
\begin{aligned}
r^{(0,1)}\left(t, t^{\prime}\right) & =L x \sin (\beta-\theta)+\frac{L^{2} t \sin (2(\beta-\theta))}{2} \\
& =L((u-a) \cos (\theta)+(v-b) \sin (\theta)) \sin (\beta-\theta)+\frac{L^{2} t \sin (2(\beta-\theta))}{2} .
\end{aligned}
$$

- If $t^{\prime}<t$, then $r\left(t, t^{\prime}\right)=\left(x+L t^{\prime} \cos (\beta-\theta)\right)(y+L t \sin (\beta-\theta))$ so that

$$
\left\{\begin{array}{l}
r^{(2,0)}\left(t, t^{\prime}\right)=0 \\
r^{(1,1)}\left(t, t^{\prime}\right)=\frac{L^{2} \sin (2(\beta-\theta))}{2} \\
r^{(0,2)}\left(t, t^{\prime}\right)=0,
\end{array}\right.
$$

and

$$
\begin{aligned}
r^{(0,1)}\left(t, t^{\prime}\right) & =L y \cos (\beta-\theta)+\frac{L^{2} t \sin (2(\beta-\theta))}{2} \\
& =L(-(u-a) \sin (\theta)+(v-b) \cos (\theta)) \cos (\beta-\theta) \frac{L^{2} t \sin (2(\beta-\theta))}{2}
\end{aligned}
$$

Finally, in both situations the covariance function $r\left(t, t^{\prime}\right)$ is continuous in $[0,1]^{2}$ and has second derivatives which are uniformly bounded for $t \neq t^{\prime}$. Then we can define the singularity function $\alpha_{i}$ of $Z$ in the situation $i, i=1,2$, defined for $t \in[0,1]$ by

$$
\alpha_{i}(t ; \beta, u, v, L)=\lim _{t^{\prime} \nearrow t} r^{(0,1)}\left(t, t^{\prime}\right)-\lim _{t^{\prime} \backslash t} r^{(0,1)}\left(t, t^{\prime}\right) .
$$

From the previous calculus we obtain

$$
\left\{\begin{array}{l}
\alpha_{1}(t ; \beta, u, v, L)=L(u-a) \sin (\beta-2 \theta)+L(v-b) \cos (\beta-2 \theta)+L^{2} t \sin (2 \beta-2 \theta) \\
\alpha_{2}(t ; \beta, u, v, L)=-L(u-a) \sin (\beta)+L(v-b) \cos (\beta) .
\end{array}\right.
$$

Note also that $\alpha_{1}$ and $\alpha_{2}$ are continuously derivable with respect to $t$ in $[0,1]$, so that they have a bounded first derivative in $[0,1]$.

For all $t$ in $[0,1]$, we denote using $V_{n, i}(t ; \beta, u, v, L)$ the quadratic variation $V_{n}(t ; \beta, u, v, L)$ in the situation $i, i=1,2$. We can now directly deduce from Perrin [10] the following result.

Theorem 3.1 For $i=1,2$, almost surely

$$
\lim _{n \rightarrow \infty} \sup _{t \in[0,1]}\left|V_{n, i}(t ; \beta, u, v, L)-\int_{0}^{t} \alpha_{i}(w ; \beta, u, v, L) d w\right|=0 .
$$


We set

$$
\begin{aligned}
I_{1}(t ; u, v, L, \beta) & =\int_{0}^{t} \alpha_{1}(w ; \beta, u, v, L) d w \\
& =((u-a) \sin (\beta-2 \theta)+(v-b) \cos (\beta-2 \theta)) L t+\frac{t^{2} L^{2}}{2} \sin (2 \beta-2 \theta),
\end{aligned}
$$

and

$$
\begin{aligned}
I_{2}(t ; u, v, L, \beta) & =\int_{0}^{t} \alpha_{2}(w ; \beta, u, v, L) d w \\
& =(-(u-a) \sin (\beta)+(v-b) \cos (\beta)) L t .
\end{aligned}
$$

\subsection{Results in the other quadrants}

Similar computations will lead to the results that we summarize in Tables 1 and 2. In Table 1, we give the singularity function for each of the quadrant in the situation $1\left(0 \leq \beta-\theta \leq \frac{\pi}{2}\right)$ and in Table 2 , we give the singularity function for each of the quadrant in the situation $2\left(-\frac{\pi}{2} \leq \beta-\theta \leq 0\right)$.

Table 1: Situation 1

\begin{tabular}{|c|c|}
\hline Quadrant & Singularity function \\
\hline$x>0$ and $y>0$ (I) & $L(u-a) \sin (\beta-2 \theta)+L(v-b) \cos (\beta-2 \theta)+L^{2} t \sin (2 \beta-2 \theta)$ \\
$x<0$ and $y>0$ (II) & $-L(u-a) \sin (\beta)+L(v-b) \cos (\beta)$ \\
$x<0$ and $y<0$ (III) & $-L(u-a) \sin (\beta-2 \theta)-L(v-b) \cos (\beta-2 \theta)-L^{2} t \sin (2 \beta-2 \theta)$ \\
$x>0$ and $y<0$ (IV) & $L(u-a) \sin (\beta)-L(v-b) \cos (\beta)$ \\
\hline
\end{tabular}

Table 2: Situation 2

\begin{tabular}{|c|c|}
\hline Quadrant & Singularity function \\
\hline$x>0$ and $y>0$ (I) & $-L(u-a) \sin (\beta)+L(v-b) \cos (\beta)$ \\
$x<0$ and $y>0$ (II) & $L(u-a) \sin (\beta-2 \theta)+L(v-b) \cos (\beta-2 \theta)+L^{2} t \sin (2 \beta-2 \theta)$ \\
$x<0$ and $y<0$ (III) & $L(u-a) \sin (\beta)-L(v-b) \cos (\beta)$ \\
$x>0$ and $y<0$ (IV) & $-L(u-a) \sin (\beta-2 \theta)-L(v-b) \cos (\beta-2 \theta)-L^{2} t \sin (2 \beta-2 \theta)$ \\
\hline
\end{tabular}

\section{Estimation of the transformation}

Let us first recall that $L, u, v$ and $\beta$ are the parameters under our control and that $a, b$ and $\theta$ are the parameters we want to estimate. For this purpose, and as previously said, we assume that the random field $Y_{\theta, a, b}$ is observed in an arbitrary non vacuous open set of $\mathbb{R}^{2}$ which does not intersect any axis of the 
canonical coordinate system. This means that $Y_{\theta, a, b}$ is observed inside one of the four quadrants of Figure 1.

As we will see in Paragraph 4.2 , the estimation of $(\theta, a, b)$ is deduced from the identification of (3) and (4) through the corresponding quadratic variations (cf. Theorem 3.1). However, as shown by Tables 1 and 2, the behavior of the quadratic variations depends on the quadrant where the random field $Y_{\theta, a, b}$ is observed. So before estimating these parameters we first have to identify the quadrant of $\left(O^{\prime}, \mathcal{X}, \mathcal{Y}\right)$ where $Y_{\theta, a, b}$ is observed.

Finally note that because $\theta$ is a unknown parameter, we are sure to be in the situation 1 only if $\beta=\frac{\pi}{2}$ and we are sure to be in the situation 2 only if $\beta=0$. This explains why identification and/or estimation are possible when $\beta$ is limited to these two values. But other choices for $\beta$ are possible and can be useful.

\subsection{Identification of the quadrant}

We use directly Tables 1 and 2 that we write again hereafter by taking explicitly $\beta=\frac{\pi}{2}$ in Table 1 and $\beta=0$ in Table 2 . We also give these Tables in terms of the limits $I_{1}(t ; u, v, L, \beta)$ and $I_{2}(t ; u, v, L, \beta)$ as defined by (3) and (4), where we take $t=1$ in both limits, $\beta=\frac{\pi}{2}$ in $I_{1}(t ; u, v, L, \beta)$ and $\beta=0$ in $I_{2}(t ; u, v, L, \beta)$. We set $I_{1}(u, v, L)=I_{1}\left(1 ; u, v, L, \frac{\pi}{2}\right)$ and $I_{2}(u, v, L)=I_{2}(1 ; u, v, L, 0)$.

Table 3: Situation 1 with $\beta=\frac{\pi}{2}$

\begin{tabular}{|c|c|}
\hline Quadrant & $I_{1}(u, v, L)$ \\
\hline$x>0$ and $y>0$ (I) & $L(u-a) \cos (2 \theta)+L(v-b) \sin (2 \theta)+\frac{L^{2}}{2} \sin (2 \theta)$ \\
$x<0$ and $y>0$ (II) & $-L(u-a)$ \\
$x<0$ and $y<0$ (III) & $-L(u-a) \cos (2 \theta)-L(v-b) \sin (2 \theta)-\frac{L^{2}}{2} \sin (2 \theta)$ \\
$x>0$ and $y<0$ (IV) & $L(u-a)$ \\
\hline
\end{tabular}

Table 4: Situation 2 with $\beta=0$

\begin{tabular}{|c|c|}
\hline Quadrant & $I_{2}(u, v, L)$ \\
\hline$x>0$ and $y>0$ (I) & $L(v-b)$ \\
$x<0$ and $y>0$ (II) & $-L(u-a) \sin (2 \theta)+L(v-b) \cos (2 \theta)-\frac{L^{2}}{2} \sin (2 \theta)$ \\
$x<0$ and $y<0$ (III) & $-L(v-b)$ \\
$x>0$ and $y<0$ (IV) & $L(u-a) \sin (2 \theta)-L(v-b) \cos (2 \theta)+\frac{L^{2}}{2} \sin (2 \theta)$ \\
\hline
\end{tabular}


From Tables 3 and 4 we deduce some properties for $I_{1}(u, v, L)$ and $I_{2}(u, v, L)$ that we gather in Table 5. As we will show in the sequel, some combinations of these properties allow us to identify the quadrant.

Table 5: Some Properties of $I_{1}(u, v, L)$ and $I_{2}(u, v, L)$

\begin{tabular}{|c|c|c|}
\hline Quadrant & $I_{1}(u, v, L)$ & $I_{2}(u, v, L)$ \\
\hline$x>0$ and $y>0$ (I) & positively quadratic in $L$ & positively linear in $L$ \\
$x<0$ and $y>0$ (II) & positively linear in $L$ & negatively quadratic in $L$ \\
$x<0$ and $y<0$ (III) & negatively quadratic in $L$ & positively linear in $L$ \\
& & \\
$x>0$ and $y<0$ (IV) & positively linear in $L$ & positively quadratic in $L$ \\
\hline
\end{tabular}

Let us give one example of identification assuming that $\theta \neq 0$. We first take $\beta=\frac{\pi}{2}$ and we use the fact that $L$ is a parameter under our control. If the limit $I_{1}(u, v, L)$ of the quadratic variation $V_{n}\left(1 ; \frac{\pi}{2}, u, v, L\right)$ depends (positively) linearly on $L$, then the quadrant is II or IV. Secondly, we take $\beta=0$ and if the limit $I_{2}(u, v, L)$ of the quadratic variation $V_{n}(1 ; 0, u, v, L)$ is positively quadratic in $L$ the quadrant is IV, otherwise the limit $I_{1}(u, v, L)$ is negatively quadratic in $L$ and the quadrant is II. Of course, this procedure is not unique, but all the procedures are consistent with each other. For instance, we could have first taken $\beta=0$.

We have just assumed that $\theta \neq 0$ but this is not a restriction. Indeed, we easily deduce from Table 5 that we can identify the angle $\theta=0$, as well as the angle $\theta=\frac{\pi}{4}$.

\section{Theorem 4.1}

$\theta=0$ if and only if $I_{1}(u, v, L)+I_{2}(u, v, L)$ is positively linear in $L$. $\theta=\frac{\pi}{4}$ if and only if $I_{1}(u, v, L)-I_{2}(u, v, L)$ is purely quadratic in $L$.

From now on, we are interested in the estimation of $(\theta, a, b)$ where $\theta \in$ ] $0, \frac{\pi}{4}[\cup] \frac{\pi}{4}, \frac{\pi}{2}[$.

\subsection{Estimation of the parameters}

As mentioned at the beginning of Section 4 , estimation of $(\theta, a, b)$ is deduced from the limits of the quadratic variations we listed in Tables 3 and 4 . So, once the quadrant can be identified, we suppose, as an example and without any restriction, that $Y_{\theta, a, b}$ is observed in an open set strictly included in the positive quadrant I. We can estimate the three unknown parameters $\theta, a$ and $b$ by using a convenient set of quadratic variations, that is a set that makes $(\theta, a, b)$ identifiable. However, there are different ways to estimate the parameters depending on the quadratic variations we choose, that is depending on the choice of the segment $[A, B]$. Nevertheless, since we have to estimate three unknown parameters $(\theta, a, b)$, we at least need a set of three quadratic variations. In fact, we decide to take four quadratic variations, and we justify our choice in the sequel. We consider $V_{n}\left(1 ; 0, u_{0}, v_{1}, L\right), V_{n}\left(1 ; \frac{\pi}{2}, u_{1}, v_{0}, L\right), V_{n}\left(1 ; \frac{\pi}{2}, u_{1}, v_{1}, L\right)$ 
and $V_{n}\left(1 ; \frac{\pi}{2}, u_{2}, v_{0}, L\right)$. From Theorem 3.1 we have almost surely, as $n$ tends to infinity

$$
\begin{aligned}
V_{n}\left(1 ; 0, u_{0}, v_{1}, L\right) & \longrightarrow L\left(v_{1}-b\right) \\
V_{n}\left(1 ; \frac{\pi}{2}, u_{1}, v_{0}, L\right) & \longrightarrow L\left(\left(u_{1}-a\right) \cos (2 \theta)+\left(v_{0}-b\right) \sin (2 \theta)\right)+\frac{L^{2}}{2} \sin (2 \theta) \\
V_{n}\left(1 ; \frac{\pi}{2}, u_{1}, v_{1}, L\right) & \longrightarrow L\left(\left(u_{1}-a\right) \cos (2 \theta)+\left(v_{1}-b\right) \sin (2 \theta)\right)+\frac{L^{2}}{2} \sin (2 \theta) \\
V_{n}\left(1 ; \frac{\pi}{2}, u_{2}, v_{0}, L\right) & \longrightarrow L\left(\left(u_{2}-a\right) \cos (2 \theta)+\left(v_{0}-b\right) \sin (2 \theta)\right)+\frac{L^{2}}{2} \sin (2 \theta) .
\end{aligned}
$$

We deduce from these convergences, the following estimators, $\hat{a}_{n}$ for $a, \hat{b}_{n}$ for $b$ and $\hat{\theta}_{n}$ for $\theta$ as follows.

$$
\begin{aligned}
& \hat{a}_{n}=u_{1}+\frac{\left(u_{2}-u_{1}\right)}{\left(v_{1}-v_{0}\right)\left(V_{n}\left(1 ; \frac{\pi}{2}, u_{2}, v_{0}, L\right)-V_{n}\left(1 ; \frac{\pi}{2}, u_{1}, v_{0}, L\right)\right)} \\
& \times\left(\left(v_{0}-v_{1}\right) V_{n}\left(1 ; \frac{\pi}{2}, u_{1}, v_{1}, L\right)\right. \\
& \left.+\left(V_{n}\left(1 ; 0, u_{0}, v_{1}, L\right)+\frac{L}{2}\right)\left(V_{n}\left(1 ; \frac{\pi}{2}, u_{1}, v_{1}, L\right)-V_{n}\left(1 ; \frac{\pi}{2}, u_{1}, v_{0}, L\right)\right)\right), \\
& \hat{b}_{n}=v_{1}-\frac{V_{n}\left(1 ; 0, u_{0}, v_{1}, L\right)}{L} \\
& \hat{\theta}_{n}=\frac{1}{2} \arctan \left(\frac{\left(V_{n}\left(1 ; \frac{\pi}{2}, u_{1}, v_{1}, L\right)-V_{n}\left(1 ; \frac{\pi}{2}, u_{1}, v_{0}, L\right)\right)}{\left(V_{n}\left(1 ; \frac{\pi}{2}, u_{2}, v_{0}, L\right)-V_{n}\left(1 ; \frac{\pi}{2}, u_{1}, v_{0}, L\right)\right)} \frac{\left(u_{2}-u_{1}\right)}{\left(v_{1}-v_{0}\right)}\right) .
\end{aligned}
$$

We also deduce the following convergence result.

Theorem 4.2 The estimators $\hat{a}_{n}, \hat{b}_{n}$ and $\hat{\theta}_{n}$ defined by (9), (10) and (11) converge almost surely to $a, b$ and $\theta$, as $n$ tends to infinity.

Proof. In this proof we mainly insist on the construction of the estimators, the convergence result follows easily. Note that this proof can be viewed as an algorithm.

- First, we directly deduce from (5) that

$$
\hat{b}_{n}=v_{1}-\frac{V_{n}\left(1 ; 0, u_{0}, v_{1}, L\right)}{L} .
$$

- Then from (6) and (8) we get almost surely, as $n$ tends to infinity

$$
V_{n}\left(1 ; \frac{\pi}{2}, u_{2}, v_{0}, L\right)-V_{n}\left(1 ; \frac{\pi}{2}, u_{1}, v_{0}, L\right) \longrightarrow L\left(u_{2}-u_{1}\right) \cos (2 \theta),
$$

so that we deduce the following estimator for $\cos (2 \theta)$ 


$$
\widehat{\cos (2 \theta})_{n}=\frac{V_{n}\left(1 ; \frac{\pi}{2}, u_{2}, v_{0}, L\right)-V_{n}\left(1 ; \frac{\pi}{2}, u_{1}, v_{0}, L\right)}{L\left(u_{2}-u_{1}\right)} .
$$

This would give an estimator of $\theta$ by using the function arccos. But $V_{n}\left(1 ; \frac{\pi}{2}, u_{2}, v_{0}, L\right)-V_{n}\left(1 ; \frac{\pi}{2}, u_{1}, v_{0}, L\right)$ does not necessarily belong to $[-1,1]$ so that $\widehat{\cos (2 \theta)})_{n}$ cannot always be defined. For preventing this shortage we estimate $\theta$ through function arctan. This is why we take four quadratic variations instead of three. More precisely, from (6) and (7) we get almost surely

$$
V_{n}\left(1 ; \frac{\pi}{2}, u_{1}, v_{1}, L\right)-V_{n}\left(1 ; \frac{\pi}{2}, u_{1}, v_{0}, L\right) \longrightarrow L\left(v_{1}-v_{0}\right) \sin (2 \theta),
$$

when $n$ tends to infinity, so that we deduce the following estimator for $\sin (2 \theta)$

$\widehat{\sin (2 \theta})_{n}=\frac{V_{n}\left(1 ; \frac{\pi}{2}, u_{1}, v_{1}, L\right)-V_{n}\left(1 ; \frac{\pi}{2}, u_{1}, v_{0}, L\right)}{L\left(v_{1}-v_{0}\right)}$.

Thus, an estimator for $\theta$ is

$$
\hat{\theta}_{n}=\frac{1}{2} \arctan \left(\frac{\widehat{\sin (2 \theta)}}{\widehat{\cos (2 \theta)})_{n}}\right) .
$$

- Finally, we deduce from (6)

$$
\hat{a}_{n}=u_{1}+\frac{\left.\left.L\left(v_{0}-\hat{b}_{n}\right) \widehat{\sin (2 \theta}\right)_{n}+\frac{L^{2}}{2} \widehat{\sin (2 \theta}\right)_{n}-V_{n}\left(1 ; \frac{\pi}{2}, u_{1}, v_{0}, L\right)}{L \operatorname{Los}(2 \theta)_{n}} .
$$

Remark 4.1 To use all the available information contained in the open set where $Y_{\theta, a, b}$ is observed, we could consider $N$ sets of four quadratic variations $\left\{V_{n}\left(1 ; 0, u_{0}^{j}, v_{1}^{j}, L\right), V_{n}\left(1 ; \frac{\pi}{2}, u_{1}^{j}, v_{0}^{j}, L\right), V_{n}\left(1 ; \frac{\pi}{2}, u_{1}^{j}, v_{1}^{j}, L\right)\right.$ and $\left.V_{n}\left(1 ; \frac{\pi}{2}, u_{2}^{j}, v_{0}^{j}, L\right)\right\}$,

with $j=1, \ldots, N$, and for a large $N$. From each set $j$, we get, in the same way as in the Proof of Theorem 4.2, the estimators $\left(\hat{\theta}_{n}^{j}, \hat{a}_{n}^{j}, \hat{b}_{n}^{j}\right)$ for $(\theta, a, b)$. Thus, we could propose a robust-like version of the estimators proposed in Theorem 4.2 as follows

$$
\begin{aligned}
& \tilde{\theta}_{n}=\frac{1}{N} \sum_{j=1}^{N} \hat{\theta}_{n}^{j} \\
& \tilde{a}_{n}=\frac{1}{N} \sum_{j=1}^{N} \hat{a}_{n}^{j} \\
& \tilde{b}_{n}=\frac{1}{N} \sum_{j=1}^{N} \hat{b}_{n}^{j} .
\end{aligned}
$$

To focus on the main ideas we do not give details in that direction. 


\section{An experimental study}

Here, we consider that the transformation from $(O, \mathcal{U}, \mathcal{V})$ to $\left(O^{\prime}, \mathcal{X}, \mathcal{Y}\right)$ is the identity function. This means that instead of $W$, we observe $Y_{\theta, a, b}$ with $\theta=0$, $a=0$ and $b=0$. Moreover we suppose that we observe $Y_{\theta, a, b}$ in the positive quadrant $\mathrm{I}$, more precisely in the square $[0,2] \times[0,2]$. We choose this framework because of the simplicity of the simulations. Indeed we first simulate a standard discrete Brownian sheet $W$ (or equivalently $Y_{\theta, a, b}$ in this framework) on the regular square grid $\left[0, \frac{1}{n}, \ldots, \frac{2 n-1}{n}, 2\right] \times\left[0, \frac{1}{n}, \ldots, \frac{2 n-1}{n}, 2\right]$ with $n=1000$ as follows

1. we simulate $4 n^{2}$ independent observations $\epsilon\left(\frac{k}{n}, \frac{l}{n}\right), 1 \leq k, l \leq 2 n$, from a standard normal distribution;

2. the values $w\left(\frac{i}{n}, \frac{j}{n}\right), 1 \leq i, j \leq 2 n$, of the Brownian sheet $W$ on the regular square grid are simply obtained as follows

$$
w\left(\frac{i}{n}, \frac{j}{n}\right)=\frac{1}{n} \sum_{k=1}^{i} \sum_{l=1}^{j} \epsilon\left(\frac{k}{n}, \frac{l}{n}\right) .
$$

Then, the unknown parameters $(\theta=0, a=0, b=0)$ are estimated with the help of the four quadratic variations introduced in Paragraph 4.2

$$
V_{n}\left(1 ; 0, u_{0}, v_{1}, L\right), V_{n}\left(1 ; \frac{\pi}{2}, u_{1}, v_{0}, L\right), V_{n}\left(1 ; \frac{\pi}{2}, u_{1}, v_{1}, L\right) \text { and } V_{n}\left(1 ; \frac{\pi}{2}, u_{2}, v_{0}, L\right)
$$

where we take in this experimental study $u_{0}=v_{0}=0, u_{1}=v_{1}=1, u_{2}=2$ and $L=1$.

Finally, to evaluate the quality of the estimators $\left(\hat{\theta}_{n}, \hat{a}_{n}, \hat{b}_{n}\right)$ for $(\theta, a, b)$ proposed in the previous Paragraph, we repeat the simulation of the discrete Brownian sheet 5000 times. For each of these repetitions, we compute the estimations of $\theta, a$ and $b$. Figure 2 represents the histogram of these 5000 values for each of the parameter. The mean and the standard error computed over the 5000 estimations are indicated above the corresponding histogram.

With respect to this simulation study, we conclude that using quadratic variations allows us to provide a satisfying estimation of the unknown canonical coordinate system of the standard Brownian sheet.

\section{Discussion}

In this Section, we wish to point out one development for extending the present work. In the work [3], we are generalizing our results to the standard nondegenerate fractional Brownian sheet, that is a centered Gaussian field $W_{H_{1}, H_{2}}=$ $\left\{W_{H_{1}, H_{2}}(x, y),(x, y) \in \mathbb{R}^{2}\right\}$ with a covariance function given by

$$
\begin{aligned}
E\left(W\left(x_{1}, y_{1}\right) W\left(x_{2}, y_{2}\right)\right)=\frac{1}{4}\left(\left|x_{1}\right|^{2 H_{1}}+\left|x_{2}\right|^{2 H_{1}}-\left|x_{1}-x_{2}\right|^{2 H_{1}}\right) \\
\quad\left(\left|y_{1}\right|^{2 H_{2}}+\left|y_{2}\right|^{2 H_{2}}-\left|y_{1}-y_{2}\right|^{2 H_{2}}\right)
\end{aligned}
$$



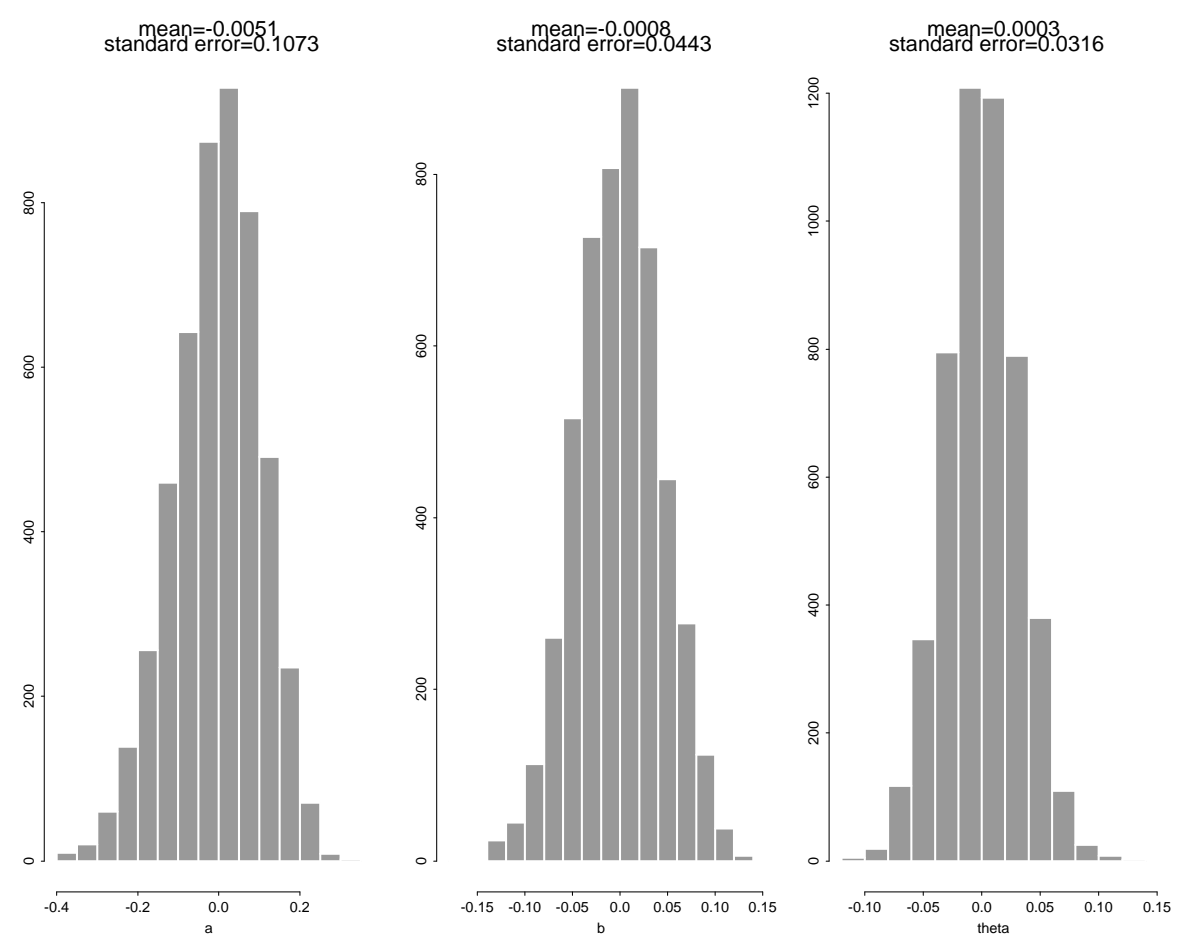

Figure 2: Histograms of the estimations of $a$ (left-hand plot), $b$ (middle plot) and $\theta$ (right-hand plot)

where $\left.\left.\left(H_{1}, H_{2}\right) \in\right] 0,1\right]^{2}$. Like here, we assume that the random field $W_{H_{1}, H_{2}}$ is observed in an arbitrarily chosen coordinate system $(O, \mathcal{U}, \mathcal{V})$, and that the transformation from $(O, \mathcal{U}, \mathcal{V})$ to $\left(O^{\prime}, \mathcal{X}, \mathcal{Y}\right)$ (where the latter is the canonical coordinate system of $W_{H_{1}, H_{2}}$ ) is a rotation through an angle $\theta$ about the origin $O$ followed by a translation of vector $(a, b)$. Thus, the definition of the standard non-degenerate fractional Brownian sheet depends on the chosen coordinate system as well as on the values of parameters $\left(H_{1}, H_{2}\right)$, unlike the standard Brownian sheet which only depends on the coordinate system (indeed for the standard Brownian sheet $H_{1}=H_{2}=\frac{1}{2}$ ).

The difficulty we are currently dealing with relies on the estimation of the parameters of the transformation $(\theta, a, b)$ together with the estimation of the parameters $\left(H_{1}, H_{2}\right)$ of the field. However the identification method we developed in the present paper can be applied to this more sophisticated problem. More precisely, quadratic variations can be useful for estimating both the coordinate system and the parameters of the random field itself, which was the original problem mentioned in the third Paragraph of the Introduction.

Moreover, in [3], we generalize the main result of Gladyshev [4] to generalized quadratic variations. Estimation of the true axes of a fractional Brownian sheet is obtained as an application of this generalization. 


\section{References}

[1] Adler, R.J. and Pyke, R. (1993). Uniform quadratic variation of Gaussian processes, Stochastic Processes and their Applications 48, 191-209.

[2] Baxter, G. (1956). A strong limit theorem for Gaussian processes, Proceedings of the American Mathematical Society 7, 522-527.

[3] Cohen, S., Guyon, X., Perrin, O. and Pontier, M. (2003). Singularity functions for fractional processes, and application to fractional Brownian sheet. Work in progress.

[4] Gladyshev, E.G. (1961). A new limit theorem for processes with Gaussian increments, Theory Probab. Appl. 6, 52-61.

[5] Guyon, X. (1987). Variations de champs gaussiens stationnaires : application à l'identification, Probability Theory and Related Fields 75, 179-193.

[6] Guyon, X. and León, J.R. (1989). Convergence en loi des H-variations d'un processus gaussien stationnaire sur $\mathbb{R}$, Annales de l'Institut Henri Poincaré 25, 265-282.

[7] Istas, J. and Lang, G. (1997). Quadratic variations and estimation of the local Hölder index of a Gaussian process, Annales de l'Institut Henri Poincaré 33 4, 407-436.

[8] León, J.R. and Ortega, J. (1989). Weak convergence of different types of variations for biparametric Gaussian processes, Colloquia Math. Soc. J. Bolyai 57, Limit theorem in Proba. and Stat. (Pecs, Hungary) pp. 349-364.

[9] Lévy, P. (1940). Le mouvement brownien plan, Amer. J. Math 62, 487-550.

[10] Perrin, O. (1999). Quadratic variation for Gaussian processes and application to time deformation, Stochastic Processes and their Applications, 82, 293-305.

[11] Sampson, P.D., Damian, D. and Guttorp, P. (2001). Advances in modeling and inference for environmental processes with nonstationary spatial covariance. In: GeoENV 2000: Geostatistics for environmental applications, P. Monestiez, D. Allard, R. Froidevaux, eds., Dordrecht: Kluwer, pp. 17-32.

[12] Sampson, P.D. and Guttorp, P. (1992). Nonparametric estimation of nonstationary spatial covariance structure, Journal of the American Statistical Association 87, 108-119. 